\title{
Gall Bladder Drainage for Acute Cholecystitis in Surgically Unfit Patients: Endoscopic Ultrasound Guided Transmural Drainage or Endoscopic Transpapillary Drainage!
}

\author{
Jimil Shah ${ }^{1}$ Surinder S. Rana ${ }^{1}$ \\ ${ }^{1}$ Department of Gastroenterology, Postgraduate Institute of \\ Medical Education and Research (PGIMER), Chandigarh, India
}

\begin{abstract}
Address for correspondence Surinder S. Rana, MD, DM, Department of Gastroenterology, Postgraduate Institute of Medical Education and Research (PGIMER), Chandigarh 160023, India (e-mail: drsurinderrana@gmail.com).
\end{abstract}

Abstract

Keywords
- gall bladder
- endosonography
- endoscopic retrograde
cholangiography
- ultrasound

Acute cholecystitis (AC) is a potentially serious condition that is associated with significant morbidity and mortality. Laparoscopic cholecystectomy is the treatment of choice of AC in surgically fit candidates. However, high-risk surgical patients with $A C$ are a therapeutic dilemma. Various alternative treatment options available include percutaneous transhepatic cholecystostomy (PTC), endoscopic ultrasound (EUS)-guided gall bladder (GB) drainage or endoscopic transpapillary drainage of GB. Due to higher complication, unplanned hospital readmission rate, risk of tube dislodgement, and high risk of recurrent acute cholecystitis associated with PTC, endoscopic drainage GB is the preferred, minimally invasive treatment option. Both endoscopic transpapillary $G B$ drainage as well as EUS-guided transmural drainage of $G B$ are effective $G B$ drainage options, but both of them have not been compared in terms of their efficacy and safety. In this news and views, we have discussed two interesting articles which have compared EUS-guided GB drainage and endoscopic transpapillary drainage.

\section{Introduction}

Acute cholecystitis (AC) is a potentially life-threatening inflammatory condition of the gall bladder (GB) which can be calculous or acalculous in nature. ${ }^{1}$ Nearly $10 \%$ of the Indian population is estimated to have gallstones, and 1 to $3 \%$ of these patients develop symptomatic gallstones. ${ }^{2}$ Mortality due to acute cholecystitis is approximately 1 to $10 \%$ and therefore the importance of treating it urgently and appropriately. ${ }^{3}$ The treatment of choice in AC is early laparoscopic cholecystectomy. However, many patients are unfit for surgical cholecystectomy either due to high risk of anesthesia/surgery or unwillingness of patient for surgical cholecystectomy. In such a scenario, it is important to drain infected GB by other modalities. Various nonsurgical therapeutic modalities like percutaneous transhepatic cholecystostomy (PTC), endoscopic ultrasound (EUS)-guided GB drainage, or endoscopic transpapillary drainage of GB have been described as effective modalities for GB drainage.
PTC is a well-established method of GB drainage. It is associated with higher technical success rate (>95\%) and higher clinical success rate (90\%). However, it is also associated with higher risk of complications like biliary peritonitis, pneumothorax, and bleeding. Moreover, risk of accidental slippage of catheter and risk of recurrent cholecystitis is as high as 10 to $15 \%{ }^{4,5}$ Studies have shown equal technical and clinical success for percutaneous or EUS-guided biliary drainage in patients with AC who are unfit for surgical cholecystectomy. However, PTC has higher complication rate, unplanned hospital readmission rate, tube dislodgement rate, and higher risk of recurrent acute cholecystitis compared with EUS-guided GB drainage group..$^{4-7}$ Endoscopic transpapillary drainage of GB is also a well-established method of GB drainage; however, there are no trials comparing EUS-guided GB drainage with transpapillary drainage. In this news and views, we have discussed two interesting articles which have compared 
EUS-guided GB drainage and endoscopic transpapillary drainage. Although both the articles are single center and retrospective in nature, the conclusion of these articles will help an endoscopist to make the correct decision with regard to the modality of GB drainage in a surgically unfit patient.

Higa JT et al have retrospectively analyzed data of 78 surgically unfit patients who were referred to their center for GB drainage. ${ }^{8}$ They have compared technical and clinical success as well as adverse events between EUS-guided GB drainage $(n=40)$ and transpapillary GB drainage $(n=38)$. The definition and classification of AC was framed according to the Tokyo guidelines. ${ }^{9}$ Technical success was defined as the ability to place transmural lumen-apposing metal stents (LAMS) under EUS guidance or transpapillary GB stent using endoscopic retrograde cholangiography (ERCP). The clinical success was defined as resolution of symptoms along with objective improvement in biochemical and radiographic findings of cholecystitis within 3 days of intervention. The reintervention was defined as any endoscopic or interventional radiology procedure for drain placement or stent-related event occurring within 30 days of the drainage procedure.

In EUS-guided intervention group, both cautery-enhanced or noncautery-enhanced 10 or $15 \mathrm{~mm}$ LAMS was used. In transpapillary stenting, once guide wire was negotiated through cystic duct, a $7 \mathrm{Fr} \times 15 \mathrm{~cm}$ double pigtail stent was placed into the GB. Amongst baseline characteristics, more patients in endoscopic transpapillary stenting group were having acute calculous cholecystitis than patients undergoing EUS-guided GB drainage (71.1 vs. $40 \% ; p=0.012$ ). On the other hand, patients undergoing EUS-guided GB drainage had more commonly acalculous cholecystitis, either malignant-related obstruction or stent-related obstruction. In both the groups, $2 / 3 \mathrm{rd}$ of the patients had moderate cholecystitis and majority of patients had obstruction at level of cystic duct. The cause of cystic duct obstruction was either stone (45 vs. $76.3 \%$ [EUS vs. transpapillary group]) or stent ( 25.0 vs. $10.5 \%$ ) or tumor (30.0 vs. $13.2 \%$ ). Technical success was higher in EUS-guided GB drainage group compared with transpapillary group ( 84.2 vs. $97.5 \%$; adjusted OR $9.83,95 \% \mathrm{CI}$ 0.93-103.86). Clinical success was also higher in EUS-guided GB drainage group compared with transpapillary group (95.0 vs. 76.3\%; adjusted OR 5.90, 95\% CI 1.18-29.41). Patients who underwent EUS-guided LAMS placement were more likely have kept stent for indefinite period than patients who had underwent transpapillary stent placement (87.2 vs. 37.5\%; OR 11.33, CI 3.48-36.91). Patients who underwent transpapillary stenting more likely underwent surgery than patients who underwent EUS-guided GB drainage (43.8 vs. 2.6\%; OR 0.034, 95\% CI 0.004-0.278). Adverse events occurred equally in both the arms (17.9\% in EUS group vs. $9.4 \%$ in transpapillary group; adjusted OR 3.04; 95\% CI, 0.58-15.78). In EUS-guided LAMS group, three cases of stent migration were observed: one intraprocedure stent migration retrieved successfully from the retroperitoneum and redeployed, one distal migration occurred 5 months postprocedure, in which stent was retrieved using antegrade double balloon enteroscopy, and one proximal migration occurred 10 months postprocedure resulting in recurrent cholecystitis treated with stent revision. Recurrent cholecystitis was more common with transpapillary drainage compared with EUS drainage (18.8 vs. 2.6\%; OR 0.094; 95\% CI, 0.011-0.81). Three patients in transpapillary group who had recurrence of $\mathrm{AC}$ were treated with EUS-guided drainage. However, other parameters like mean duration of antibiotic use, median length of postprocedure hospital stay, and median postprocedure pain score was similar in both the groups. Three patients in the EUS group had significant ascites for which authors had aspirated ascites under EUS guidance and continued paracentesis on a weekly basis for 4 weeks to facilitate cholecysto-duodenal fistula formation. Authors have also concluded that, ideally, transpapillary stenting should be the first line of treatment modality in patients with ascites. Only in case of failure of transpapillary drainage, one should attempt EUS-guided GB drainage.

In pancreatic fluid collections drained by LAMS, recent studies have shown adverse effects of indwelling LAMS for more than 3 weeks with development of pseudoaneurysm and risk of "buried stent." However, ideal time of removal of LAMS used for GB drainage is not well-defined at present. In this study, authors have shown that 18 patients had long term follow-up of indwelling LAMS ( $>1$ year) without any major side effect. However, at the same time, authors have also suggested that $7 \mathrm{~F}^{*} 3 \mathrm{~cm}$ double pigtail plastic stent can be used to replace the LAMS in order to reduce chances of injury to contralateral GB wall. The authors concluded that in patients who are surgical candidates requiring only temporary measures to drain GB or patients with significant ascites, transpapillary drainage should be considered as the first-line treatment option. However, in patients with stent-related cystic duct obstruction or malignant obstruction or patients who are poor surgical candidates, EUS-guided GB drainage can be considered as first-line treatment option in view of higher technical and clinical success and lower risk of recurrent cholecystitis.

Oh et al performed retrospective analysis of 172 patients with AC undergoing EUS-guided cholecystostomy or endoscopic transpapillary cholecystostomy. ${ }^{10}$ Definition and classification of AC was framed according to Tokyo guidelines. All included patient had failed conservative medical management for at least 24 hours. EUS-guided drainage was preferred in patients with malignant cystic duct obstruction, bile duct cancer, and cystic duct obstruction due to metallic stent placement or unsuccessful/infeasible transpapillary drainage. Endoscopic transpapillary drainage was performed when obstruction occurred due to cystic duct stone or associated common bile duct (CBD) stone. Technical success was defined as ability to place transmural metallic stent (BONA-AL stent; Standard Sci-Tech Inc, Seoul, Korea) under EUS guidance or transpapillary GB stent (7F stent) using ERCP. Clinical success was defined as resolution of symptoms along with normalization of laboratory tests. Recurrence of cholecystitis was defined as the recurrence of typical symptoms with characteristic imaging findings.

In their study, 96 patients underwent endoscopic transpapillary drainage and 76 patients underwent EUS-guided GB drainage. Seven out of 16 patients who failed transpapillary drainage cross over to EUS-guided GB drainage, 
making it 83 patients in EUS-guided GB drainage group. In the transpapillary group, more patients had CBD stone (29.2 vs. $7.2 \% ; p<0.01$ ) and less patients had malignancy (67.5 vs. $20.8 \% ; p<0.01$ ) as an etiology compared with EUS-guided GB drainage. EUS-guided GB drainage group had more technical (98.8 vs. $83.3 \% ; p<0.01)$ and clinical success rate (98.8 vs. $82.3 \% ; p<0.01$ ) compared with endoscopic transpapillary group by intention to treat analysis. However, there was no statistical difference in clinical success rate by per-protocol analysis between both the groups ( 100 vs. $98.8 \% ; p=0.49$ ). In the EUS-guided GB drainage group, the procedure failed in one patient (1.2\%) because of accidental loss of the guide wire during stent placement. In the endoscopic transpapillary group, the procedure failed in 16 patients (16.7\%) because of selective cystic duct cannulation failure $(n=12)$ or nonvisualization of the cystic duct because of cystic duct obstruction $(n=4)$. Procedure time ( $18.3 \pm 4.9$ vs. $19.5 \pm 9.6$ minute; $p=0.31$ ) and procedure-related adverse events ( 7.2 vs. $9.4 \% ; p=0.75$ ) were similar in both the groups. In the EUS-guided GB drainage group, pneumoperitoneum occurred in three patients (3.6\%), duodenal perforation in one patient (1.2\%), and recurrent biliary pain in two patients (2.4\%). In patients with recurrent biliary pain, additional double pigtail plastic stents were placed to maintain the stent patency. In the endoscopic transpapillary group, eight patients experienced post-ERCP pancreatitis which improved after conservative treatment. Recurrent biliary pain occurred in one patient after 3 days of procedure. In this study, only five patients underwent elective laparoscopic cholecystectomy. Recurrent cholecystitis was more common in endoscopic transpapillary group versus EUS-guided GB drainage group (17.4 vs. 3.9\%; $p=0.05$ ). In EUS-guided GB drainage group, three patients experienced recurrent cholecystitis because of stent strut fracture $(n=2)$ or stent occlusion because of food impaction $(n=1)$, while in the endoscopic transpapillary group, 10 patients experienced recurrent cholecystitis because of distal stent migration $(n=6)$ or stent occlusion ( $n$ $=4$ ). The authors concluded that although both modalities of GB drainage are safe and effective, EUS-guided GB drainage may be more suitable due to better clinical success and lesser risk of recurrent $\mathrm{AC} .^{10}$

\section{Commentary}

Acute cholecystitis, either calculus or non-calculus, is associated with higher morbidity and mortality. Treatment of choice in such a scenario is usually surgical cholecystectomy. However, in up to $10 \%$ of patients, surgical removal of GB is not feasible due to various reasons. In such a scenario, if patient is fit for endoscopy, endoscopic GB drainage is preferred over percutaneous transhepatic drainage due to lower risk of complications and lesser risk of recurrent AC. In endoscopic modalities, there are two different modalities: EUS-guided GB drainage and endoscopic transpapillary drainage. These studies, although retrospective, showed that EUS-guided biliary drainage has better technical and clinical success with lesser risk of recurrent cholecystitis. These studies have not conducted subgroup analysis of clinical success and risk of recurrent cholecystitis according to the etiology of AC. Although several studies have used LAMS for EUS-guided GB drainage, there are still certain unanswered questions like ideal time for removal of LAMS or exchanging it with plastic stent. Also, unique difficulties that surgeon might face due to LAMS during elective cholecystectomy are still not defined. Siddiqui et al compared PTC $(n=146)$, EUS-guided GB drainage ( $n=124)$, and endoscopic transpapillary drainage $(n=102)$ retrospectively. They also found that endoscopic transpapillary drainage had lesser technical and clinical success rates compared with other two modalities. EUS-guided GB drainage group had shorter hospital stay and less long-term adverse events rate compared with PTC and transpapillary group. ${ }^{11}$

To conclude, both modalities of endoscopic GB drainage are associated with high technical and clinical success rates. Endoscopic transpapillary drainage should be preferred in patients with benign obstruction or in presence of CBD stone. It should be ideally used as a temporary bridge therapy in patients who are good surgical candidates. However, due to high risk of recurrent $A C$, its use as a definitive long-term modality to drain GB should be avoided. EUS-guided GB drainage should preferentially be used in presence of malignant cystic duct obstruction or cystic duct obstruction due to metallic stent. Due to lower risk of recurrent AC, it can be used as a definitive measure to drain GB in a poor surgical candidate patient.

\section{Conflict of Interest}

None declared.

\section{References}

1 Friedman GD. Natural history of asymptomatic and symptomatic gallstones. Am J Surg 1993;165(4):399-404

2 Tandon RK. Prevalence and type of biliary stones in India. World J Gastroenterol 2000;6:4-5

3 Kimura Y, Takada T, Strasberg SM, et al. TG13 current terminology, etiology, and epidemiology of acute cholangitis and cholecystitis. J Hepatobiliary Pancreat Sci 2013;20(1):8-23

4 Teoh AYB, Serna C, Penas I, et al. Endoscopic ultrasound-guided gallbladder drainage reduces adverse events compared with percutaneous cholecystostomy in patients who are unfit for cholecystectomy. Endoscopy 2017;49(2):130-138

5 Irani S, Ngamruengphong $\mathrm{S}$, Teoh $\mathrm{A}$, et al. Similar efficacies of endoscopic ultrasound gallbladder drainage with a lumen-apposing metal stent versus percutaneous transhepatic gallbladder drainage for acute cholecystitis. Clin Gastroenterol Hepatol 2017;15(5):738-745

6 Tyberg A, Saumoy M, Sequeiros EV, et al. EUS-guided versus percutaneous gallbladder drainage: isn't it time to convert? J Clin Gastroenterol 2018;52(1):79-84

7 Jang JW, Lee SS, Song TJ, et al. Endoscopic ultrasound-guided transmural and percutaneous transhepatic gallbladder drainage are comparable for acute cholecystitis. Gastroenterology 2012;142(4):805-811

8 Higa JT, Sahar N, Kozarek RA, et al. EUS-guided gallbladder drainage with a lumen-apposing metal stent versus endoscopic transpapillary gallbladder drainage for the treatment of acute cholecystitis (with videos). Gastrointest Endosc 2019;90(3):483-492 
9 Hirota M, Takada T, Kawarada Y, et al. Diagnostic criteria and severity assessment of acute cholecystitis: Tokyo Guidelines. J Hepatobiliary Pancreat Surg 2007;14(1):78-82

10 Oh D, Song TJ, Cho DH, et al. EUS-guided cholecystostomy versus endoscopic transpapillary cholecystostomy for acute cholecystitis in high-risk surgical patients. Gastrointest Endosc 2019;89(2):289-298

11 Siddiqui A, Kunda R, Tyberg A, et al. Three-way comparative study of endoscopic ultrasound-guided transmural gallbladder drainage using lumen-apposing metal stents versus endoscopic transpapillary drainage versus percutaneous cholecystostomy for gallbladder drainage in high-risk surgical patients with acute cholecystitis: clinical outcomes and success in an International, Multicenter Study. Surg Endosc 2019;33(4):1260-1270 